RHIC-PH-1

\title{
REPORT OF TASR FORCE FOR \\ RELATIVISTIC HEAVY ION PHYSICS
}

T. Ludlam and A. Schwarzschild

Brookhaven National Laboratory

August 30,1983 


\section{BROOKHAVEN NATIONAL LABORATORY \\ MEMORANDUM}

DATE: $\quad$ August 30,1983

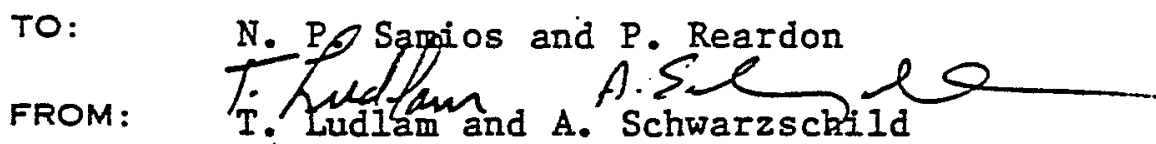

SUBJECT: Report of Task Force for Relativistic Heavy Ion Physics

We are reporting on the conclusions of the Task Force regarding accelerator specifications for a Heavy Ion Collider Facility and interim Research and Development program to properly address the 1mportant Physics questions of this field. The Task Force met for three days of deliberation on August 22-24, 1983. Stimulated most recently by the NSAC decision to recognize such a facility as its "highest priority new program" and by the laboratory's possible interest in such a new large endeavor, it was relatively easy to assemble a knowledgeable group of physicists to participate in this study on very short notice. A distinguished group comprising theorists, experimentalists and accelerator scientists from both the nuclear and high energy physics communities participated. Representation from other labs engaged in or considering such a program (LBL, ORNL, CERN) was included.

The assembled group expressed great enthusiasm for the physics discussed. Generaliy there was impatience to get on with the program in the U.S. The Task Force encourages the laboratory to design and propose a Relativistic Heavy Ion Collider quickly, and to support, with all available resources, the interim program to accelerate ions from the Tandem in the AGS. There was a distinct sense of the high energy physics participants that this program should not be the exclusive domain of "Nuclear Physics". We believe that is a positive attitude and that the cooperation of both. communities (as evidenced by this small group) is appropriate to the Importance of the physics and the magnitude of the task.

The momentum and excitement generated in this small group will continue at the Quark Matter 1983 Conference at BNL in one month. The issues addressed here will be further refined and exposed to the larger world community at that time. We believe that significant community support will be forthcoming for a program at BNL in this field. 
The participants in the task force deliberations are as follows:

The members of the Task Force were:
G. Baym, Univ, of Illinois
L. Madansky, J.Hopkins Univ
J.D. Bjorken, FNAI
C. Gelbke, MSU
H. Gutbrod, GSI
A. Rerman, MIT
C. Leemann, LBL
A. Mueller, Columbia Univ
I. Otterlund, Univ of Lund
A. Ruggiero, FNAL
L. Schroeder, LBL
G. Young, ORNL
W. Willis, CERN

BNL members:

M. Barton

T. Ludlam, Co-chairman

0 . Eansen

R. Palmer

R. Foley

M. Sakitt

S. Rahana

A. Schwarzschild, Co-chairman

Additional participants active in the discussions of the working sessions were:

D. Barton, BNL

E. Courant, BNL

H. Hahn, BNL

S. Lindenbaum, BNL

F. Paige, BNL
N. P. Samios, BNL

J. Sandweiss, Yale Univ.

T. L. Trueman, BNL

H. Wegner, BNL 
The task force discussion was guided by the list of questions given in Table $I$. These issues were addressed both in the full panel - following presentations of specific points of view by various panel members - and in small working groups. The agenda for each of the three days' discussions Is attached. We present here the principal conclusions reached by the panel on each of the questions. The conclusions regarding the parameters of a heavy ion collider are sumarized in Table II.

\section{BASIC MACHINE PARAMETERS FOR A HIGH ENERGY HEAVY ION COLLIDER}

\section{ENERGY OF BEAMS:}

It was generally agreed that the role of a collider is to achieve large energy densities in a central rapidity region of small baryon number density. Requiring a central region spanning two units of rapidity, and taking into account uncertainties in the size of the fragmentation regions in heavy ion collisions, the panel concluded that the machine should reach beam energies (GeV/nucleon) in the range $50+50$ to $100+100$.

It was pointed out that this range of energy is also indicated by the fact that fixed target heavy ion beams at the CERN SPS can achieve energies equivalent to a $10+10 \mathrm{GeV} /$ nucleon collider. Though such a program has not been officially approved, it is technically straightforward and thought likely by the end of the decade. Any new undertaking should aim for a significantly higher energy region.

A further point made by the panel was that a collider dedicated to this physics should span a range of energies, extending low enough to explore the on-set of nuclear transparency: roughly corresponding to beam energies of $5+5 \mathrm{GeV} / \mathrm{nucleon}$.

\section{RANGE OF ION MASSES :}

Most predictions for the interesting physics phenomena seem to saturate as $\mathrm{A}^{1 / 3}$. However, certain phenomena related to large distance behavior of QCD may give rise to much stronger functions of $A$, and require the largest nuclei. The minimum requirement for exploring the regime of truly heavy lons is $A=100$, and it is expected that a collider dedicated to heavy ion physics would ultimately reach $A=200$ (e.g. Au, $\mathrm{Pb}$ ). At this stage no apparent advantage is seen in accelerating heavier ions. 
The machine should accelerate light ions as well, including protons.

\section{LUMINOSITY}

The Iuminosity requirements for initial experiments are thought to be rather modest. Assuming a cross section of $10 \mathrm{mb}$ for the interesting (head-on) collisions, a luminosity $I=10^{26} \mathrm{~cm}^{-2} \mathrm{sec}^{-1}$ would provide one such event per second. There are currentiy no strong theoretical arguments which place a premium on significantly higher event rates. The recomendation is thus that a minimum $I=10^{25} \mathrm{~cm}^{-2} \mathrm{sec}^{-1}$ (at the upper end of the energy range) be attainable initially. Nonetheless, it is deemed likely that the early, exploratory measurements will lead to a demand for higher event rates. The machine should be designed to attain an ultimate luminosity of $I \geq 10^{28} \mathrm{~cm}^{-2} \mathrm{sec}^{-1}$. At this level, owing to the high final state multiplicities, high particle rates will begin to degrade detector lifetime and performance.

At a certain level the coulomb interaction cross sections leading to photodisintegration will. reduce the lifetime of the stored beams to an impractically small value. Such ultimate limits to the luminosity have been roughly estimated to set in at $L \gtrsim 10^{28} \mathrm{~cm}^{-2} \mathrm{sec}^{-1}$, but this is a subject that urgently needs more study.

\section{ASMMETRIC OPERATION}

Two possible modes of asymmetric collider operation were discussed:

(i) Different ion masses in the two rings; 1.e., Iight ion on heavy Ion.

(ii) Different energies in the two rings.

Mode ( 1 ) is thought to be an important option for some aspects of the physics program. For example, comparison of P-A with AA collisons may be crucial to the understanding of basic interaction models. This mode of 
operation does not put severe constraints on the design of a collider, unlike mode (ii). Operation with asymmetrlc energies does not broaden the range of physics parameters, but is primarily a matter of gaining easier access to certain kinematic regions in the final state. It was agreed that this is not a mode of operation that should be stressed in the design of a machine.

\section{INTERSECTION REGIONS}

A minimum of 3 intersection regions is required for a facility to carry out the range of physics envisioned. Up to 6 IRs could be adequately exploited.

The length of straight section in each IR along which detectors can be arrayed must allow for the forward instrumentation required for sensitive event selection on impact parameter, and the study of particle production in the fragmentation regions. This will call for a free space of at least $\pm 10 \mathrm{~m}$. Special intersection regions may emphasize these forward measurements, as is the case with the small angle hall in the CBA design.

The intersection regions should be well spaced around the machine to reduce the backgrounds from beam halo produced in upstream crossings.

An important source of such background is the Coulomb dissociation cross section, which arises from virtual photons created in the E-M field of relativistic ions passing near each other, leading to $(\gamma, n)$ or fission processes. Since the Coulomb excitation probability goes as $\mathrm{z}^{2}$, these cross sections become huge for heavy ion collisions. Values of -100 Barnshave been estimated, which would not only induce severe backgrounds, but would reduce the beam lifetime to a few hours at $\mathrm{L} \simeq 10^{28} \mathrm{~cm}^{-2} \mathrm{sec}^{-1}$. These processes are amenable to calculation, and the Task Force urges an immediate effort to gain a quantitative understanding of the limitations which they impose. 
II. EXPERIMENTS AND DETECTOR REQUIREMENTS

LARGE FACILITIES AND "SMALL" EXPERIMENTS

It is expected that there will be at least two large-solid-angle spectrometers, of which at least one will be operated in a facility-like manner to allow access for the traditional small nuclear physics group.

It is important that large detector systems not be designed in such a way as to be too narrowly focussed on preconceived notions (e.g. our present ones) regarding the important signatures and properties of new phenomena. It will be well for such detectors to come on in a phased way, so that there is some flexibility to respond to surprises in the first round of measurements.

The possibility of reusing an existing detector system for at least one of the large spectrometers should be considered. Detectors from the ISR, or from $e^{+} e^{-}$colliders could provide the basic elements for a heavy ion collider. Any such detector would require new solutions to handle the high track densities of the interesting nucleus-nucleus collistons.

At least one intersection region should be devoted to small solid-angle experiments. The anticipated physics - including many of the more speculative issues - allows for a great variety of small experiments with relatively rapid turn-around.

NUMBER OF USERS

A collider facility of the sort being discussed here requires a certain minimum number of users if it is to generate an effective program of physics. The Task Force, considering the size of experiments and experimental programs at existing colliding beam facilities, concluded that the minimum number in this case is about 300 physicists. To set the scale, this number corresponds to about $8 \%$ of the total U.S. nuclear and high energy physics community. 
The time table of the NSAC recomendation may be part of a long range plan in which major construction funding for a heavy ion collider would follow the construction of the recentiy-approved $4 \mathrm{GeV}$ electron facility. On such a schedule the heavy ion machine would be completed early in the next decade. This time scale could be significantly advanced if funding could be started sooner. The Task Force felt that an involvement by high energy physics was not only desirable, but essential for the success of this undertaking - and such an involvement could be the means for an earlier start on construction.

By 1986 it should be possible to provide nuclear beams in the U.S. with masses up to 32 and energy of $15 \mathrm{GeV} /$ nucleon for fixed target experiments at the AGS. This corresponds to an equivalent collider energy (GeV/nucleon) of about 2+2, opening to exploration a region of energy about $5 x$ that of the Bevalac. Experiments at these energies can study extreme states of high baryon density up to the limits imposed by nuclear transparency. Aside from the a priori potential for new discoveries in this energy range, such experiments will provide crucial information for constraining the models upon which estimates of behavior at very high energy are based. These experiments will also serve to begin the process of assembling the necessary large user groups for a subsequent collider program, as well as establishing bridges between nuclear and high energy physics groups.

The Task Force concluded that the proposed scheme for injecting lons up to mass 32 directly from the BNL tandem accelerator to the AGS should be implemented as soon as possible - both to provide a fixed target program competitive with the CERN PS experiments by 1986, and to allow early machine studies of the AGS performance as a potential collider injector.

Detector development is another critical element of the intermediate program, and requires immediate attention. Efficient tracking and particle identification at the anticipated particle densities of heary ion collisions is at the limit of the present state of the art for high energy detectors. This area is the subject of intense R\&D effort by high energy physics and should be given additional support from nuclear physics. 
IV. SPECIFIC MACHINE QUESTIONS : IMPLICATIONS FOR BNL

The accelerator experts found this workshop most beneficial. There was a broad discussion of accelerator issues and problems and an enlightening discussion of the opproaches to these problems being pursued at the various laboratorles. These discussions made clear that there are significant economic advantages at Brookhaven that should enable BNL to advance a most attractive proposal for a collider. The Tandem injection program should be started as soon as possible so that some hands on expertise is developed even if this is not the ultimate injector. There are.a number of options that must be sketched out in design and costs established for comparisons. These include the large aperture CBA magnet, use of Fermilab magnets, superferric magnets (both warm and cold iron), room temperature magnets, and SSC magnets. For the AGS there are a number of injector options which must be studied. There are also a host of scaling rules, beam dynamics issues, interation point questions, etc., that are more generic than BNL's own plans and all of the laboratories need to be involved in study of these questions. The physics community needs more input on the tradeoffs between various machine parameters and costs. There was some interest in accelerator experts from varlous laboratories having small informal meetings several times a year for review of progress on these points. BNL should take intiative in organizing such meetings. 
Table I

Questions Addressed by the Task Force

I. Physics Questions and Basic Machine Parameters:

- Discovery thresholds in energy and $A$

- Energy of beams

- Range of ion masses

- Luminosity

- Number of intersection regions

- Size of intersection regions

II. Detector Requirements:

- How many experiments?

-How many large facilities?

-How many physicists?

III. Specific Machine Questions

- Source/injector

- Cholce of magnets

-R\&D requirements

- Cost estimates

IV. Other Questions

-Time Scale:

What is NSAC's time table?

Other possibilities?

-Intermediate Program

How to bring together the user community, and establish a connection between nuclear and particle groups?

-Experiments at LBL

-Experiments at CERN

-Experiments at AGS

-Detector R\&D 
Parameters of a High Energy Heavy Ion Collider

Energy of Beams (GeV/amu):

-At least $50+50$, covering a range of energies starting as low as $5+5$.

Range of Ion Masses:

-A > 100 initialiy; ultimately $A \geq 200$.

- Iight ions also, Inclualng protons.

Luminosity:

$\cdot L \geq 10^{25} \mathrm{~cm}^{-2} \mathrm{sec}^{-1}$ initially; ultimately reaching $L \geqslant 10^{28} \mathrm{~cm}^{-2} \mathrm{sec}^{-1}$.

Intersections:

-3-6 Intersection Regions

- Free space along beams at least $\pm 10 \mathrm{~m}$

Detectors and Experiments:

-At least one or two large, facility-like detectors with $4 \pi$ coverage. -Many opportunitles for small solid-angle experiments.

-Expect a user community of $\geqq 300$ physicists. 
TASK FORCE ON ULTRA RELATIVISTIC HEAVY ION PHYSICS

Program of Meetings

Monday, August 22, 1983

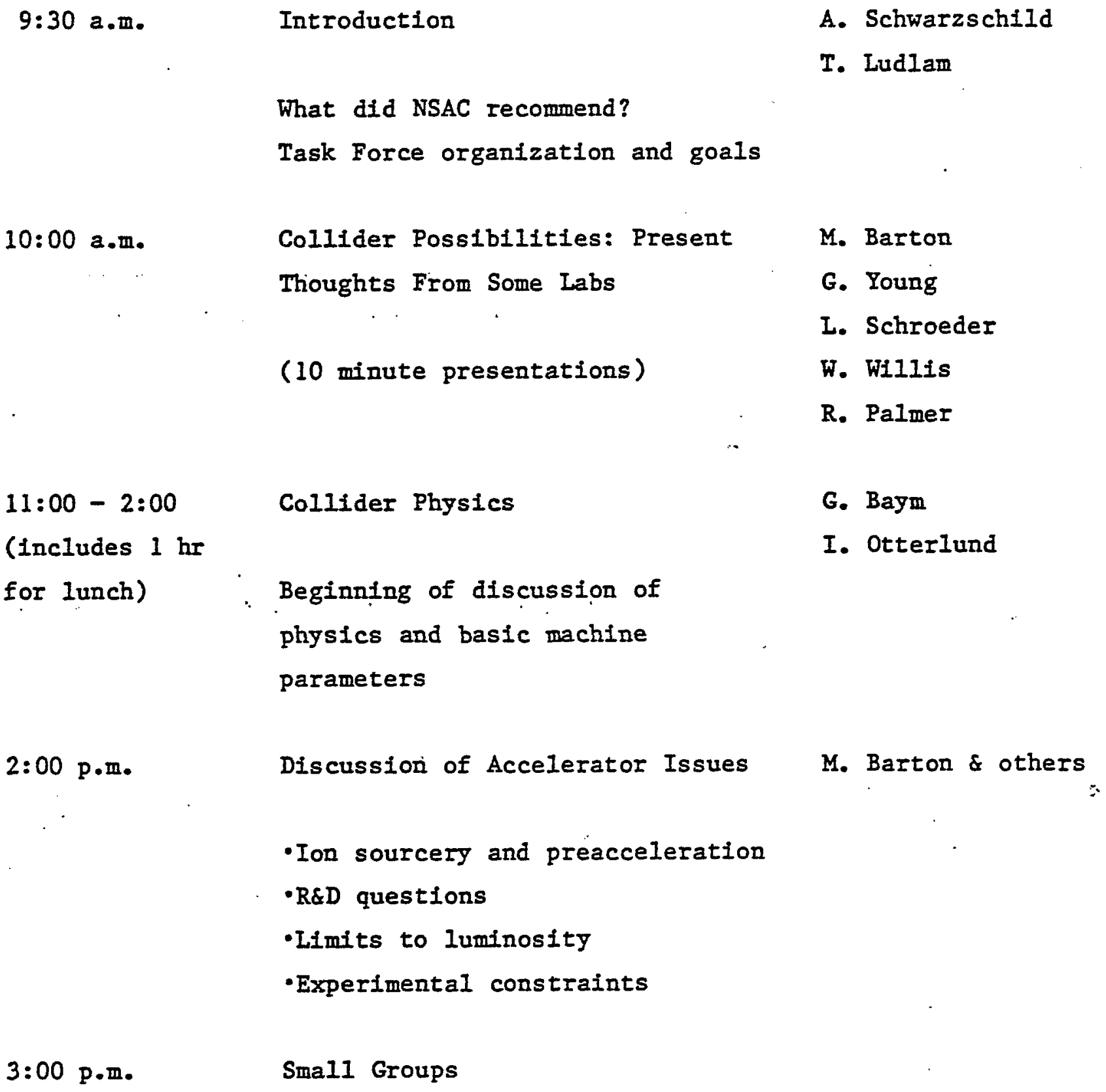

- Machine topics

- Physics topics

- Detectors, etc. 
Tuesday, August 23, 1983

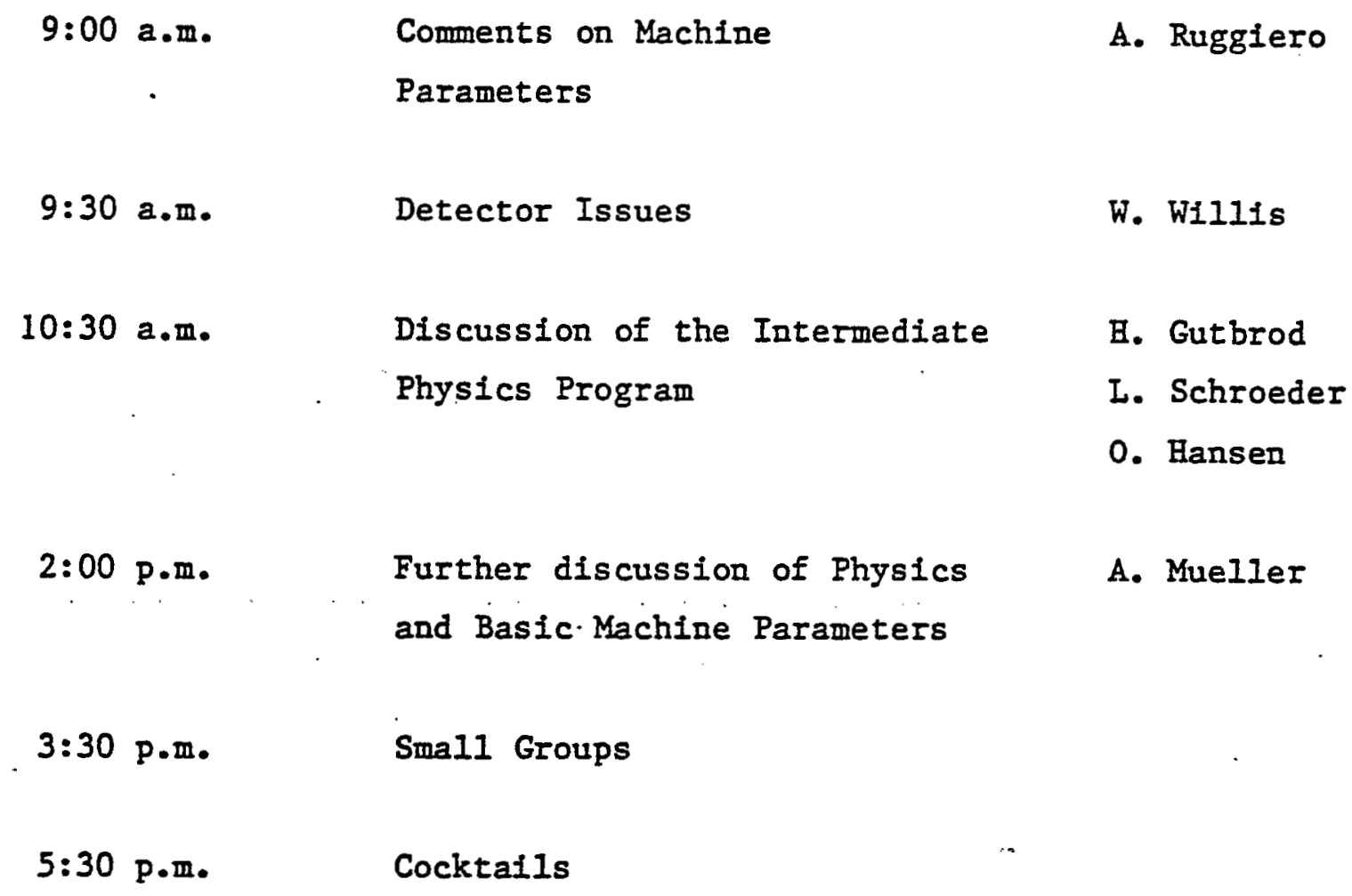

Wednesday, August 24,1983

9:00 a.m. Small Groups

10:00 a.m. Continued Discussion of Physics J.D. Bjorken and Basic Machine Parameters

1:15 p.m. Summary of Task Force Conclusions T. Ludlam

2:30 p.m. Adjourn 